\title{
THE REGULARITY OF DUNFORD-PETTIS OPERATORS
}

\author{
JAMES R. HOLUB
}

(Communicated by John B. Conway)

\begin{abstract}
Let $\lambda$ denote a symmetric, solid Banach sequence space having $\left\{e_{i}\right\}_{i=1}^{\infty}$ as a symmetric basis and considered as a Banach lattice with order defined coordinatewise. A complete description of the relationship between regular and Dunford-Pettis operators $T: L^{1}[0,1] \rightarrow \lambda$ is given. The results obtained complete earlier work of Gretsky and Ostroy and of the author in this area.
\end{abstract}

1. Introduction. If $X$ and $Y$ are Banach spaces, a bounded linear operator $T: X \rightarrow Y$ is called a Dunford-Pettis operator (or, as we write, a D-P operator) if $T$ maps weakly convergent sequences in $X$ to norm convergent ones in $Y$. In the case where $X$ and $Y$ are Hilbert spaces such operators were once called "completely continuous", but the terminology gradually disappeared in favor of the notion of a compact operator (with which it agrees when the domain of the operator is a reflexive Banach space). In general, however, compactness of an operator is a more restrictive condition, a fact which is rather dramatically apparent in the case of operators defined on $l^{1}$, all of which are D-P. The term "Dunford-Pettis operator" was introduced by Grothendieck [8] in view of the pioneering work of Dunford and Pettis [3] in investigating the properties of such operators on $L^{1}$ and $C(S)$-spaces. Due to its importance for various applications subsequent work has continued to focus on understanding the structure of $\mathrm{D}-\mathrm{P}$ operators from the space $L^{1}[0,1]$ to various specific, as well as general, Banach spaces (e.g. $[\mathbf{1}, \mathbf{2}, \mathbf{5}, \mathbf{6}, \mathbf{7}, \mathbf{9}, \mathbf{1 1}, \mathbf{1 3}, \mathbf{1 4}$, 16]).

A particular case in point is the paper of Gretsky and Ostroy [6] on D-P operators from $L^{1}[0,1]$ to certain Banach lattices. Motivated by considerations of mathematical economics [5] they showed that every regular operator (i.e. a difference of positive operators) from $L^{1}[0,1]$ to a Banach lattice having an "order compatible" Schauder basis is a D-P operator; in particular (their case of greatest interest) every regular operator from $L^{1}[0,1]$ to $c_{0}$ is D-P. In a recent paper [9] the author showed the converse of this last result is also true, so the D-P operators from $L^{1}[0,1]$ to $c_{0}$ are precisely the regular operators.

The question which now arises is whether the converse of the Gretsky-Ostroy theorem is true in general. In particular, suppose $\lambda$ is a symmetric, solid, Banach sequence space (i.e., if $\left\{a_{i}\right\}_{i=1}^{\infty} \in \lambda$ then $\left\{a_{\pi(i)}\right\}_{i=1}^{\infty} \in \lambda$ for any permutation $\pi$, and if $\left|b_{i}\right| \leq\left|a_{i}\right|$ for all $i$ then $\left(b_{i}\right) \in \lambda$ ). Suppose, too, that the unit vectors $\left\{e_{j}\right\}_{j=1}^{\infty}$ in $\lambda$, defined by $e_{j}=\left\{\delta_{i, j}\right\}_{i=1}^{\infty}$, form a symmetric basis for $\lambda$ and that the order

Received by the editors September 30, 1987.

1980 Mathematics Subject Classification (1985 Revision). Primary 47B37, 47B55; Secondary 47A65.

Key words and phrases. Dunford-Pettis operator, regular operator. 
on $\lambda$ is defined by $\left\{a_{i}\right\}_{i=1}^{\infty} \leq\left\{b_{i}\right\}_{i=1}^{\infty} \Leftrightarrow a_{i} \leq b_{i}$ for all $i$. Then $\left\{e_{j}\right\}_{j=1}^{\infty}$ is an order compatible basis for $\lambda$, so every regular operator $T: L^{1}[0,1] \rightarrow \lambda$ is D-P. The question we consider is the converse: Is every $\mathrm{D}-\mathrm{P}$ operator $T: L^{1}[0,1] \rightarrow \lambda a$ regular operator?

The purpose of the present paper is to give a complete solution to this problem. The interesting aspect of the solution itself is the fact that the positive answer obtained for the case $\lambda=c_{0}$ mentioned above is completely atypical, in the following sense: If $\lambda=\lambda^{x x}$ then every bounded linear operator $T: L^{1}[0,1] \rightarrow \lambda$ is regular, while if $\lambda \neq \lambda^{x x}$ (the case of $c_{0}$ ) then the only time every D-P operator $T: L^{1}[0,1] \rightarrow$ $\lambda$ is regular is when $\lambda=c_{0}$; hence the result proved in [9] is more fortuitous than characteristic. Finally, we show that for any $\lambda$ every weakly compact operator $T: L^{1}[0,1] \rightarrow \lambda$ is regular, a satisfying result pertinent to this questions since every such weakly compact operator is a D-P operator $[10$, p. 182].

2. If $E$ and $F$ are Banach spaces we denote the set of all bounded linear operators from $E$ to $F$ by $\mathscr{L}(E, F)$. If $E$ is a Banach space and $x \in E$, we denote the norm of $x$ in $E$ by $\|x\|_{E}$. In the case where $E$ is $L^{p}$ or $l^{p}$ for $1 \leq p \leq+\infty$, we will write $\|x\|_{E}=\|x\|_{p}$. Throughout the paper $\lambda$ will always denote a symmetric, solid, Banach sequence space in which the unit vectors $\left\{e_{j}\right\}_{j=1}^{\infty}$ form a symmetric Schauder basis and in which the order is defined coordinatewise (as we outlined in $\S 1)$. In particular, then, $\left\{e_{j}\right\}_{j=1}^{\infty}$ is an order compatible basis for $\lambda$, so every regular operator $T: L^{1}[0,1] \rightarrow \lambda$ is a D-P operator.

Recall that the Köthe dual of $\lambda$ is the sequence space

$$
\lambda^{x}=\left\{\left\{b_{i}\right\}_{i=1}^{\infty}\left|\sum_{i=1}^{\infty}\right| a_{i}|| b_{i} \mid<+\infty \text { for all }\left\{a_{i}\right\}_{i=1}^{\infty} \text { in } \lambda\right\} .
$$

By the Uniform Boundedness Principle and the fact that $\left\{e_{i}\right\}_{i=1}^{\infty}$ is a basis for $\lambda$ it follows that $\lambda^{x}$ may be identified with the dual space $\lambda^{*}$ of $\lambda$. Hence we consider $\lambda^{x}$ as a Banach space with norm defined by

$$
\left\|\left\{b_{i}\right\}_{i=1}^{\infty}\right\|_{\lambda^{x}}=\sup \left\{\sum_{i=1}^{\infty}\left|a_{i}\right|\left|b_{i}\right| \mid\left\|\left\{a_{i}\right\}_{i=1}^{\infty}\right\|_{\lambda} \leq 1\right\} .
$$

Note that unless $\left\{e_{i}\right\}_{i=1}^{\infty}$ is a basis for $\lambda^{x}$ we will not generally have $\lambda^{x x}=\lambda^{* *}$, although we always have $\lambda \subset \lambda^{x x}$. Those space $\lambda$ for which $\lambda^{x x}=\lambda$ are called perfect. (For more detailed information on such matters see $\S 30$ of the book Topological vector spaces. I, by G. Köthe, Springer-Verlag, 1969.)

We begin with the following observation about operators from $L^{1}[0,1]$ to $\lambda$ which will be seen to lead naturally to our main results.

THEOREM 1. If $T: L^{1}[0,1] \rightarrow \lambda$ is any bounded linear operator, then

$$
\left\{\left\|T^{*} e_{i}\right\|_{1}\right\}_{i=1}^{\infty} \in \lambda^{x x} .
$$

If $\left\{\left\|T^{*} e_{i}\right\|_{1}\right\}_{i=1}^{\infty} \in \lambda$, then $T$ is a $D$-P operator.

ProOF. Since $T \in \mathscr{L}\left(L^{1}[0,1], \lambda\right), T^{*}: \lambda^{x} \rightarrow L^{\infty}[0,1]$ is both norm and weak*continuous. If $\left\{a_{i}\right\}_{i=1}^{\infty} \in \lambda^{x}$ with $\left\|\left\{a_{i}\right\}_{i=1}^{\infty}\right\|_{\lambda^{x}} \leq 1$ then $\sum_{i=1}^{\infty} \varepsilon_{i} a_{i} e_{i}$ is weak ${ }^{*}$ convergent to $\left\{\varepsilon_{i} a_{i}\right\}_{i=1}^{\infty}$ for every sequence $\left\{\varepsilon_{i}\right\}_{i=1}^{\infty}$ with $\left|\varepsilon_{i}\right|=1$, and $\left\|\sum_{i=1}^{\infty} \varepsilon_{i} a_{i} e_{i}\right\|_{\lambda^{x}} \leq 1$ 
also. Therefore $\sum_{i=1}^{\infty} \varepsilon_{i} a_{i} T^{*} e_{i}$ is weak ${ }^{*}$-convergent in $L^{\infty}[0,1]$ and

$$
\left\|\sum_{i=1}^{\infty} \varepsilon_{i} a_{i} T^{*} e_{i}\right\|_{\infty} \leq\left\|T^{*}\right\| .
$$

From the definition of the norm in $L^{\infty}[0,1]$ it follows that $\sum_{i=1}^{\infty}\left|a_{i}\right|\left|T^{*} e_{i}(t)\right| \leq\left\|T^{*}\right\|$ a.e. in $[0,1]$, so by the Dominated Convergence Theorem the series $\sum_{i=1}^{\infty}\left|a_{i}\right|\left|T^{*} e_{i}\right|$ converges in $L^{1}[0,1]$ and $\left\|\sum_{i=1}^{\infty}\left|a_{i}\left\|T^{*} e_{i} \mid\right\|_{1} \leq\left\|T^{*}\right\|\right.\right.$. But then $\left.\left.\sum_{i=1}^{\infty}\right| a_{i} \mid\right\| T^{*} e_{i} \|_{1} \leq$ $\left\|T^{*}\right\|$ for all $\left(a_{i}\right) \in \lambda^{x}$ with $\left\|\left(a_{i}\right)\right\|_{\lambda^{x}} \leq 1$, and by definition it follows that

$$
\left\{\left\|T^{*} e_{i}\right\|_{1}\right\}_{i=1}^{\infty} \in \lambda^{x x} .
$$

Now suppose $\left\{\left\|T^{*} e_{i}\right\|_{1}\right\}_{i=1}^{\infty}$ is actually in $\lambda \subset \lambda^{x x}$. It is well known that $T: L^{1}[0,1] \rightarrow \lambda$ is a D-P operator if and only if $T \circ i: L^{\infty}[0,1] \rightarrow L^{1}[0,1] \rightarrow$ $\lambda$ is compact [1] (where $i: L^{\infty}[0,1] \rightarrow L^{1}[0,1]$ is the canonical injection map). Since $\left\{\left\|T^{*} e_{i}\right\|_{1}\right\} \in \lambda$ and $\left\{e_{i}\right\}_{i=1}^{\infty}$ is a basis for $\lambda,\left\|\sum_{i=n}^{\infty}\right\| T^{*} e_{i}\left\|_{1} e_{i}\right\|_{\lambda} \rightarrow 0$ as $n \rightarrow \infty$. Therefore $\left|\sum_{i=n}^{\infty} a_{i}\left\|T^{*} e_{i}\right\|_{1}\right| \rightarrow 0$ uniformly over $\left\|\left\{a_{i}\right\}_{i=1}^{\infty}\right\|_{\lambda^{x}} \leq 1$, so given $\varepsilon>0$ choose $n$ so that $\left|\sum_{i=n}^{\infty} a_{i}\left\|T^{*} e_{i}\right\|_{1}\right|<\varepsilon$ for all $\left\|\left(a_{i}\right)\right\|_{\lambda^{x}}$ $\leq 1$. Then $\left|\sum_{i=n}^{\infty} a_{i}\left\langle T^{*} e_{i}, g\right\rangle\right|<\varepsilon$ whenever $\left\|\left(a_{i}\right)\right\|_{\lambda^{x}} \leq 1$ and $\|g\|_{\infty} \leq 1$, or $\left|\left\langle\sum_{i=n}^{\infty}\left\langle T \circ i(g), e_{i}\right\rangle e_{i},\left\{a_{i}\right\}_{i=1}^{\infty}\right\rangle\right|<\varepsilon$ whenever $\left\|\left(a_{i}\right)\right\|_{\lambda^{x}} \leq 1$ and $\|g\|_{\infty} \leq 1$. That is, $\left\|\sum_{i=n}^{\infty}\left\langle T \circ i(g), e_{i}\right\rangle e_{i}\right\|_{\lambda} \rightarrow 0$ as $n \rightarrow \infty$, uniformly over $g$ in the unit ball of $L^{\infty}[0,1]$, so $\left\{T \circ i g \mid\|g\|_{\infty} \leq 1\right\}$ is conditionally compact in $\lambda[4$, p. 260]. Therefore $T \circ i$ is compact and it follows that $T$ is a D-P operator.

It is natural to ask whether the converse of the last assertion of Theorem 1 is true, and hence whether it is possible to characterize the D-P operators from $L^{1}[0,1]$ to $\lambda$ by the condition that $\left\{\left\|T^{*} e_{i}\right\|\right\}_{i=1}^{\infty} \in \lambda$. According to the first part of Theorem 1 this will be true whenever $\lambda=\lambda^{x x}$, and it was shown in [9] to also be true when $\lambda=c_{0}$. It turns out, however, that this is not always the case. In fact, (as in the case $\lambda=c_{0}$ in [9]) the condition $\left\{\left\|T^{*} e_{i}\right\|_{1}\right\}_{i=1}^{\infty} \in \lambda$ actually characterizes the regularity of operators from $L^{1}[0,1]$ to $\lambda$.

THEOREM 2. $T: L^{1}[0,1] \rightarrow \lambda$ is regular $\Leftrightarrow\left\{\left\|T^{*} e_{i}\right\|_{1}\right\}_{i=1}^{\infty} \in \lambda$.

PROOF. $(\Rightarrow)$ To show that a regular operator $T: L^{1}[0,1] \rightarrow \lambda$ has the property that $\left\{\left\|T^{*} e_{i}\right\|_{1} \|\right\} \in \lambda$, we need only show this is true whenever $T \geq 0$. Therefore, suppose $T: L^{1}[0,1] \rightarrow \lambda$ is a positive operator. Then $T$ is a D-P operator [6], so $T \circ i: L^{\infty}[0,1] \rightarrow L^{1}[0,1] \rightarrow \lambda$ is compact [1]. We showed in Theorem 1 that then $\left|\sum_{i=n}^{\infty}\left\langle T^{*} e_{i}, g\right\rangle a_{i}\right| \rightarrow 0$ as $n \rightarrow \infty$, uniformly over $g \in L^{\infty}[0,1]$ with $\|g\|_{\infty} \leq 1$ and $\left\{a_{i}\right\}_{i=1}^{\infty} \in \lambda^{x}$ with $\left\|\left\{a_{i}\right\}_{i=1}^{\infty}\right\|_{\lambda^{x}} \leq 1$, and since $T \geq 0$ we have $T^{*} e_{i} \geq 0$ a.e. in $[0,1]$ for all $i=1,2, \ldots$ Given any $\varepsilon>0$ choose $N$ so that if $n \geq N$ then $\left|\sum_{i=n}^{\infty}\left\langle T^{*} e_{i},|g|\right\rangle\right| a_{i} \|<\varepsilon$ whenever $\|g\|_{\infty} \leq 1$ and $\left\|\left\{a_{i}\right\}_{i=1}^{\infty}\right\|_{\lambda^{x}} \leq 1$. That is, $\left|\left\langle\sum_{i=n}^{\infty}\left|a_{i}\right| T^{*} e_{i},|g|\right\rangle\right|<\varepsilon$ for all $\|g\|_{\infty} \leq 1$ and all $\left\|\left\{a_{i}\right\}_{i=1}^{\infty}\right\|_{\lambda^{x}} \leq 1$, so $\left\|\sum_{i=n}^{\infty}\left|a_{i}\right| T^{*} e_{i}\right\|_{1}<\varepsilon$ whenever $n \geq N$ and $\left\|\left\{a_{i}\right\}_{i=1}^{\infty}\right\|_{\lambda^{x}} \leq 1$. But since $T^{*} e_{i} \geq 0$ a.e. this says that $\sum_{i=n}^{\infty}\left|a_{i}\right|\left\|T^{*} e_{i}\right\|_{1}<\varepsilon$ whenever $\left\|\left(a_{i}\right)\right\|_{\lambda^{x}} \leq 1$ and $n \geq N$, so $\sum_{i=1}^{\infty}\left\|T^{*} e_{i}\right\|_{1} e_{i}$ converges in $\lambda$; that is, $\left\{\left\|T^{*} e_{i}\right\|_{1}\right\}_{i=1}^{\infty} \in \lambda$.

$(\Leftarrow)$ On the other hand, suppose $T: L^{1}[0,1] \rightarrow \lambda$ and $\left\{\left\|T^{*} e_{i}\right\|_{1}\right\}_{i=1}^{\infty} \in \lambda$. To show $T$ is regular we first show that we can define a bounded linear operator $|T|: L^{1}[0,1] \rightarrow \lambda$ by $|T|(f)=\left\{\left\langle f,\left|T^{*} e_{i}\right|\right\rangle\right\}_{i=1}^{\infty}$. To do this, let $\varepsilon>0$ be given, let $f \in L^{1}[0,1]$, and choose $g \in L^{\infty}[0,1]$ so that $\|f-g\|_{1}<\varepsilon / 2\|T\|$. Then for any 
$n \geq 1$ and any $\left\{a_{i}\right\}_{i=1}^{\infty} \in \lambda^{x}$ we have

$$
|| \sum_{i=n}^{\infty} a_{i}\left\langle f,\left|T^{*} e_{i}\right|\right\rangle|\leq| \sum_{i=n}^{\infty}\left|a_{i} \|\left\langle f-g,\left|T^{*} e_{i}\right|\right\rangle\right||+| \sum_{i=n}^{\infty}\left|a_{i}\right|\left\langle g,\left|T^{*} e_{i}\right|\right\rangle \mid .
$$

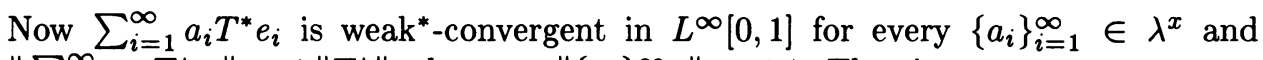
$\left\|\sum_{i=1}^{\infty} a_{i} T^{*} e_{i}\right\|_{\infty} \leq\left\|T^{*}\right\|$ whenever $\left\|\left\{a_{i}\right\}_{i=1}^{\infty}\right\|_{\lambda^{x}} \leq 1$. That is,

$$
\operatorname{ess} \sup \left|\sum_{i=1}^{\infty} \varepsilon_{i} a_{i} T^{*} e_{i}(t)\right| \leq\left\|T^{*}\right\|
$$

for all $\left\|\left\{a_{i}\right\}\right\|_{\lambda^{x}} \leq 1$ and for all $\left\{\varepsilon_{i}\right\}$ with $\left|\varepsilon_{i}\right|=1$; it follows that $\left\|\sum_{i=1}^{\infty}\left|a_{i}\left\|T^{*} e_{i} \mid\right\|_{\infty}\right.\right.$ $\leq\left\|T^{*}\right\|=\|T\|$ for all such $\left\{a_{i}\right\} \in \lambda^{x}$, and hence that $\left|\sum_{i=n}^{\infty}\right| a_{i}\left|\left\langle\left|T^{*} e_{i}\right|, f-g\right\rangle\right| \leq$ $\|f-g\|_{1}\|T\|<\varepsilon / 2$ whenever $\left\|\left\{a_{i}\right\}_{i=1}^{\infty}\right\|_{\lambda^{x}} \leq 1$, and for all $n$. Moreover, since $\left\{\left\|T^{*} e_{i}\right\|_{1}\right\}_{i=1}^{\infty} \in \lambda$ there is an integer $N$ such that if $n \geq N$ then $\left\|\sum_{i=n}^{\infty}\right\| T^{*} e_{i}\left\|e_{i}\right\|_{\lambda}$ $<\varepsilon / 2\|g\|_{\infty}$. Therefore if $n \geq N$ and $\left\|\left\{a_{i}\right\}\right\|_{\lambda^{x}} \leq 1$ it follows that

$$
\left|\sum_{i=n}^{\infty}\right| a_{i}\left|\left\langle\left|T^{*} e_{i}\right|, g\right\rangle\right| \leq\|g\|_{\infty} \sum_{i=n}^{\infty}\left|a_{i}\right|\left\|T^{*} e_{i}\right\|_{1}<\|g\|_{\infty} \frac{\varepsilon}{2\|g\|_{\infty}}=\frac{\varepsilon}{2},
$$

so if $n \geq N$ we have, by the above, that $\left|\sum_{i=n}^{\infty} a_{i}\left\langle f,\left|T^{*} e_{i}\right|\right\rangle\right|<\varepsilon$ for all $\left\|\left\{a_{i}\right\}_{i=1}^{\infty}\right\|_{\lambda^{x}}$ $\leq 1$. Hence the sequence $\left\{\left\langle\left|T^{*} e_{i}\right|, f\right\rangle\right\}_{i=1}^{\infty}$ is in $\lambda$ for all $f \in L^{1}[0,1]$, so the operator $|T|: L^{1}[0,1] \rightarrow \lambda$ for which $|T|(f)=\left\{\left\langle\mid T^{*} e_{i}, f\right\rangle\right\}_{i=1}^{\infty}$ is well defined and bounded.

Now clearly $|T| \geq T$ and $|T| \geq-T$ (since if $f \geq 0$ in $L^{1}[0,1]$ then

$$
\left|\left\langle T f, e_{i}\right\rangle\right|=\left|\left\langle T^{*} e_{i}, f\right\rangle\right|=\left|\int_{0}^{1} T^{*} e_{i}(t) f(t) d t\right| \leq \int_{0}^{1}\left|T^{*} e_{i}\right|(t) f(t) d t=\left\langle\left|T^{*} e_{i}\right|, f\right\rangle
$$

for all $i)$, so we have $T=((|T|+T) / 2)-((|T|-T) / 2)$, a difference of positive operators, and $T$ is regular.

COROLlARY. If $T: L^{1}[0,1] \rightarrow \lambda$ is a bounded linear operator and $\lambda^{x x}=\lambda$, then $T$ is regular and a $D-P$ operator.

We see in Corollary 1 the essential simplicity of the case $\lambda=\lambda^{x x}$, while if $\lambda \neq \lambda^{x x}$ the relationship between regular operators and D-P operators becomes more interesting. In all cases regularity implies the D-P property [6], and when $\lambda=c_{0}$ it is known that the converse also holds [9]. Surprisingly, this turns out to be the only such case (i.e. where $\lambda \neq \lambda^{x x}$ ) for which this is true.

THEOREM 3. If $\lambda \neq \lambda^{x x}$ then every $D-P$ operator $T: L^{1}[0,1] \rightarrow \lambda$ is a regular operator $\Leftrightarrow \lambda=c_{0}$.

ProOF. As we have already mentioned, if $\lambda=c_{0}$ then every D-P operator is regular.

Suppose, then, that $\lambda \neq \lambda^{x x}$ and that $\lambda \neq c_{0}$. To show there is a D-P operator which is not regular we need only demonstrate (by Theorem 2) a D-P operator $T: L^{1}[0,1] \rightarrow \lambda$ for which $\left\{\left\|T^{*} e_{i}\right\|_{1}\right\}_{i=1}^{\infty} \notin \lambda$.

We first note that since $\lambda \neq c_{0}$ it follows that $\lambda^{x x} \neq l^{\infty}$. Otherwise, if $\left\{c_{i}\right\}_{i=1}^{\infty} \in$ $l^{\infty}$ then $\left\{c_{i}\right\}_{i=1}^{\infty} \in \lambda^{x x}$ so $\sum_{i=1}^{\infty}\left|c_{i}\left\|b_{i} \mid \leq\right\|\left\{c_{i}\right\}_{i=1}^{\infty} \|_{\lambda^{x x}}\right.$ whenever $\left\|\left\{b_{i}\right\}_{i=1}^{\infty}\right\|_{\lambda^{x}} \leq 1$; in particular this would be true when $c_{i}=1$ for all $i$, implying $\left\{b_{i}\right\}_{i=1}^{\infty} \in l^{1}$ for 
all $\left\{b_{i}\right\}_{i=1}^{\infty} \in \lambda^{x}$ and hence that $\lambda^{x}=l^{1}$ (since $l^{1} \subset \lambda^{x}$ in all cases). But then whenever $\left\{c_{i}\right\}_{i=1}^{\infty} \in c_{0}$ and $p<q$

$$
\begin{aligned}
\left\|\sum_{i=p}^{q} c_{i} e_{i}\right\|_{\lambda} & =\sup \left\{\sum_{i=p}^{q}\left|c_{i}\left\|b_{i}||\right\|\left\{b_{i}\right\}_{i=1}^{\infty} \|_{\lambda^{x}} \leq 1\right\}\right. \\
& \leq \sup \left\{\sum_{i=p}^{q}\left|c_{i}\left\|b_{i} \mid\right\|\left\{b_{i}\right\}_{i=1}^{\infty} \|_{1} \leq K\right\}\right.
\end{aligned}
$$

for some constant $k$, (since $\lambda^{x}$ is isomorphic to $l^{1}$ by the above). Since this last is $\leq K \sup _{i \geq p}\left|c_{i}\right| \rightarrow 0$ as $p \rightarrow \infty$ it follows that $\left\{c_{i}\right\}_{i=1}^{\infty} \in \lambda$ and since $\lambda \subset c_{0}$ (always) we have $\lambda=c_{0}$, a contradiction. Therefore, it must be that $\lambda^{x x} \neq l^{\infty}$. From this we also see that $\lambda^{x x}$ must be a subset of $c_{0}$. If not, there is a sequence $\left\{a_{i}\right\}_{i=1}^{\infty}$ in $\lambda^{x x}$ but not in $c_{0}$, and hence an $\varepsilon>0$ and a subsequence $\left\{a_{i_{k}}\right\}_{k=1}^{\infty}$ of $\left\{a_{i}\right\}_{i=1}^{\infty}$ for which $\left|a_{i_{k}}\right| \geq \varepsilon$ for all $k$. Since $\lambda^{x x}$ is both symmetric and solid the sequence $\left\{\left|a_{i_{1}}\right|,\left|a_{i_{2}}\right|, \ldots\right\}$ is also in $\lambda^{x x}$ and hence so is any bounded sequence $\left\{b_{j}\right\}_{j=1}^{\infty}$ since we can write $\left\{b_{j}\right\}_{j=1}^{\infty}$ as the sequence

$$
\left\{\frac{b_{1}}{\left|a_{i_{1}}\right|} a_{i_{1}}, \frac{b_{2}}{\left|a_{i_{2}}\right|} a_{i_{2}}, \ldots, \frac{b_{k}}{\left|a_{i_{k}}\right|} a_{i_{k}}, \ldots\right\}, \quad \text { where } \sup _{k} \frac{\left|b_{k}\right|}{\left|a_{i_{k}}\right|} \leq \frac{1}{\varepsilon} \sup _{k}\left|b_{k}\right|<+\infty .
$$

That is, $l^{\infty} \subset \lambda^{x x}$ and since the reverse inclusion always holds we would have $l^{\infty}=\lambda^{x x}$, a contradiction to the above result.

Now let $\left\{r_{n}\right\}_{n=1}^{\infty}$ denote the set of Rademacher functions on $[0,1][15$, p. 396]. It is well known that these functions have the property that $\left\|r_{n}\right\|_{\infty}=\left\|r_{n}\right\|_{1}=1$, $n=1,2,3, \ldots$, and $\left\{r_{n}\right\}_{n=1}^{\infty}$ is an orthonormal sequence in $L^{2}[0,1]$. Choose any sequence $\left\{a_{n}\right\}_{n=1}^{\infty}$ in $\lambda^{x x}$ but not in $\lambda$, so as we noted above $\left\{a_{n}\right\}_{n=1}^{\infty} \in c_{0}$. For any function $g \in L^{2}[0,1]$ the sequence $\left\{\left\langle r_{n}, g\right\rangle\right\}_{n=1}^{\infty} \in l^{2} \subset c_{0}$, so since $\left\|r_{n}\right\|_{\infty}=1$ for all $n$ it follows by a standard approximation argument that for any $f \in L^{1}[0,1]$ the sequence $\left\{\left\langle r_{n}, f\right\rangle\right\}_{n=1}^{\infty} \in c_{0}$. Hence the sequence $\left\{a_{n}\left\langle r_{n}, f\right\rangle\right\}_{n=1}^{\infty} \in \lambda$ for all $f \in L^{1}[0,1]$, since

$$
\begin{aligned}
\left\|\sum_{n=p}^{q} a_{n}\left\langle r_{n}, f\right\rangle e_{n}\right\|_{\lambda} & =\sup \left\{\sum_{n=p}^{q}\left|a_{n}\left\|\left\langle r_{n}, f\right\rangle\right\| b_{n}\right| \mid\left\|\left\{b_{n}\right\}_{n=1}^{\infty}\right\|_{\lambda^{x}} \leq 1\right\} \\
& \leq \sup _{n \geq p}\left|\left\langle r_{n}, f\right\rangle\right| \sup \left\{\sum_{n=p}^{q}\left|a_{n}\left\|b_{n}||\right\|\left\{b_{n}\right\}_{n=1}^{\infty} \|_{\lambda^{x}} \leq 1\right\}\right. \\
& \leq \sup _{n \geq p}\left|\left\langle r_{n}, f\right\rangle\right| \cdot\left\|\left\{a_{n}\right\}_{n=1}^{\infty}\right\|_{\lambda^{x x}} \rightarrow 0 \text { as } p \rightarrow \infty,
\end{aligned}
$$

and so we can define a bounded linear operator $T: L^{1}[0,1] \rightarrow \lambda$ by

$$
T f=\sum_{n=1}^{\infty} a_{n}\left\langle r_{n}, f\right\rangle e_{n}
$$

Clearly $T^{*} e_{n}=a_{n} r_{n}$ for $n$, so $\left\{\left\|T^{*} e_{n}\right\|_{1}\right\}_{n=1}^{\infty}=\left\{\left|a_{n}\right|\right\}_{n=1}^{\infty} \notin \lambda$, and by Theorem 2 it follows that $T$ is not regular. To see that $T$ is a D-P operator, recall that if $i: L^{2}[0,1] \rightarrow L^{1}[0,1]$ is the injection map, then $T$ is a D-P operator $\Leftrightarrow T \circ i: L^{2}[0,1] \rightarrow L^{1}[0,1] \rightarrow \lambda$ is compact [1], and this last is true $\Leftrightarrow$ 
$\sum_{n=1}^{\infty}\left\langle T \circ i(f), e_{n}\right\rangle e_{n}$ converges in $\lambda$ uniformly over $f \in L^{2}[0,1]$ with $\|f\|_{2} \leq 1[\mathbf{4}$, p. 260].

If $f \in L^{2}[0,1]$ and $\|f\|_{2} \leq 1$, then for any $N$ we have

$$
\left\|\sum_{n=N}^{\infty}\left\langle T \circ i(f), e_{n}\right\rangle e_{n}\right\|_{\lambda}=\left\|\sum_{n=N}^{\infty} a_{n}\left\langle r_{n}, f\right\rangle e_{n}\right\|_{\lambda}=\sup _{\left\|\left\{b_{n}\right\}\right\|_{\lambda} x \leq 1}\left|\sum_{n=N}^{\infty}\right| a_{n}\left\|b_{n}\right\|\left\langle r_{n}, f\right\rangle \mid .
$$

Since $\left\{\left\{\left\langle r_{n}, f\right\rangle\right\}_{n=1}^{\infty} \mid\|f\|_{2} \leq 1\right\}$ is precisely the unit ball in $l^{2}$ (recall $\left\{r_{n}\right\}$ is an O.N. sequence in $\left.L^{2}[0,1]\right)$ it follows that

$$
\begin{aligned}
\sup _{\|f\|_{2} \leq 1}\left\|\sum_{n=N}^{\infty} a_{n}\left\langle r_{n}, f\right\rangle e_{n}\right\|_{\lambda} & \leq \sup _{\left\|\left\{b_{n}\right\}\right\|_{\lambda} \leq 1}\left\|\sum_{n=N}^{\infty} a_{n} b_{n} e_{n}\right\|_{2} \\
& \leq\left[\sup _{n \geq N}\left|a_{n} \| b_{n}\right|\right]^{1 / 2} \sup _{\left\|\left\{b_{n}\right\}\right\|_{\lambda} \leq 1}\left[\sum_{n=N}^{\infty}\left|a_{n} \| b_{n}\right|\right]^{1 / 2} \\
& \leq \sup _{n \geq N}\left|a_{n}\right|^{1 / 2}\left\|\left\{a_{n}\right\}\right\|_{\lambda}^{1 / 2}
\end{aligned}
$$

where this goes to zero as $N \rightarrow \infty$ since $\left\{a_{n}\right\} \in c_{0}$. Hence $\left\|\sum_{n=N}^{\infty} a_{n}\left\langle r_{n}, f\right\rangle e_{n}\right\|_{\lambda} \rightarrow$ 0 uniformly over $\|f\|_{2} \leq 1$ and, as we remarked above, it follows that $T$ is a D-P operator which is not regular.

The results given here (along with the Gretsky-Ostroy Theorem) provide a complete description of the relationship between $\mathrm{D}-\mathrm{P}$ operators and regular operators from $L^{1}[0,1]$ to $\lambda$; in particular, when $\lambda \neq \lambda^{x x}$ and $\lambda \neq c_{0}$ there always exist D-P operators from $L^{1}[0,1]$ to $\lambda$ which fail to be regular. In contrast we now show that in all cases every weakly compact operator is regular. The significance of this result in the context of those obtained previously lies in the fact that every weakly compact operator $T: L^{1}[0,1] \rightarrow \lambda$ is a D-P operator $[10, p .182]$, thereby illustrating the fundamental difference between weakly compact and D-P operators in the case where $\lambda \neq \lambda^{x x}$.

THEOREM 4. Every weakly compact operator from $L^{1}[0,1]$ to $\lambda$ is regular.

PROOF. Let $T: L^{1}[0,1] \rightarrow \lambda$ be a weakly compact operator. According to Theorem 2,T is regular if and only if $\left\{\left\|T^{*} e_{i}\right\|_{1}\right\}_{i=1}^{\infty} \in \lambda$, while by Theorem 1 $\left\{\left\|T^{*} e_{i}\right\|_{1}\right\}_{i=1}^{\infty} \in \lambda^{x x}$ in any case.

Suppose $\left\{\left\|T^{*} e_{i}\right\|_{1}\right\}_{i=1}^{\infty} \in \lambda^{x x}$, but is not in $\lambda$. Then $\sum_{i=1}^{\infty} b_{i}\left\|T^{*} e_{i}\right\|_{1}$ converges pointwise, but not uniformly, over vectors $\left\{b_{i}\right\}_{i=1}^{\infty}$ is the unit ball of $\lambda^{x}$. Hence there is some $\varepsilon>0$ and an increasing sequence $\left\{p_{k}\right\}_{k=1}^{\infty}$ of integers for which $\left\|\sum_{i=p_{k}+1}^{p_{k+1}}\right\| T^{*} e_{i}\left\|_{1} e_{i}\right\|_{\lambda} \geq \varepsilon, k=1,2, \ldots$; correspondingly, there is a sequence of unit vectors $\left\{b_{i}^{(k)}\right\}_{i=1}^{\infty}, k=1,2, \ldots$, in $\lambda^{x}$ for which $\sum_{i=p_{k}+1}^{p_{k+1}}\left|b_{i}^{(k)}\right|\left\|T^{*} e_{i}\right\|_{1} \geq \varepsilon$ for all $k$.

It is well known that the injection mapping $i: L^{\infty}[0,1] \rightarrow L^{1}[0,1]$ is weakly compact and hence a D-P operator $\left[10\right.$, p. 182], so if $\left\{\sum_{i=p_{k}+1}^{p_{k+1}}\left|b_{i}^{(k)}\right|\left|T^{*} e_{i}\right|\right\}_{k=1}^{\infty}$ were weakly convergent to zero in $L^{\infty}[0,1]$ then it would converge to zero in $L^{1}[0,1]$; but this would say $\left\{\sum_{i=p_{k}+1}^{p_{k+1}}\left|b_{i}^{(k)}\right|\left\|T^{*} e_{i}\right\|_{1}\right\}_{k=1}^{\infty} \rightarrow 0$, a contradiction to the above. Hence it must be that $\left\{\sum_{i=p_{k}+1}^{p_{k+1}}\left|b_{i}\right|\left|T^{*} e_{i}\right|\right\}_{k=1}^{\infty}$ is not weakly convergent to zero in $L^{\infty}[0,1]$. Now $L^{\infty}[0,1]$ is order-isomorphic to a space $C(S)$ of continuous functions 
on some compact Hausdorff space $S\left[\mathbf{4}\right.$, p. 445]. If, under this isometry, $T^{*} e_{i}$ corresponds to $f_{i} \in C(S)$, then $\left|T^{*} e_{i}\right|$ corresponds to $\left|f_{i}\right|$ and by the above the sequence $\left\{\sum_{i=p_{k}+1}^{p_{k+1}}\left|b_{i}^{(k)} \| f_{i}\right|\right\}$ does not converge weakly to zero in $C(S)$. Consequently there is some point $s_{0} \in S$ so that $\left\{\sum_{i=p_{k+1}}^{p_{k+1}}\left|b_{i}^{(k)} \| f_{i}\left(s_{0}\right)\right|\right\}$ does not converge to zero [4, p. 265]. Choosing numbers $\varepsilon_{i}^{(k)}$ so that $\varepsilon_{i}^{(k)} b_{i}^{(k)} f_{i}\left(s_{0}\right)=\left|b_{i}^{(k)}\right|\left|f_{i}\left(s_{0}\right)\right|$ for all $i$ and $k$ we then see that $\left\{\sum_{i=p_{k}+1}^{p_{k+1}} \varepsilon_{i}^{(k)} b_{i}^{(k)} f_{i}\right\}_{k=1}^{\infty}$ does not converge weakly to zero in $C(S)$. However the sequence $\left\{\sum_{i=p_{k}+1}^{p_{k+1}} \varepsilon_{i}^{(k)} b_{i}^{(k)} e_{i}\right\}_{k=1}^{\infty}$ is weak ${ }^{*}$-convergent to zero in $\lambda^{x}$, so since $T^{*}$ is weakly compact the sequence $\left\{\sum_{i=p_{k}+1}^{p_{k+1}} \varepsilon_{i}^{(k)} b_{i}^{(k)} T^{*} e_{i}\right\}_{k=1}^{\infty}$ must converge weakly to zero in $L^{\infty}[0,1]$, implying $\left\{\sum_{i=p_{k}+1}^{p_{k+1}} \varepsilon_{i}^{(k)} b_{i}^{(k)} f_{i}\right\}_{k=1}^{\infty}$ converges weakly to zero in $C(S)$, and we reach a contradiction. Therefore it must be that $\sum_{i=1}^{\infty} b_{i}\left\|T^{*} e_{i}\right\|_{1}$ converges uniformly over $\left\|\left\{b_{i}\right\}\right\|_{\lambda^{x}}<1$, hence that $\left\{\left\|T^{*} e_{i}\right\|_{1}\right\}_{i=1}^{\infty} \in$ $\lambda$, and $T$ is regular.

REMARK. The converse of Theorem 4 is, in general, not true. For example, if $f_{n}=\chi_{[0,1 / n]}$ for $n=1,2, \ldots$ then $\left\{f_{n}\right\}_{n=1}^{\infty}$ is weak ${ }^{*}$-convergent to zero in $L^{\infty}[0,1]$ so $T=\sum_{n=1}^{\infty} f_{n} \otimes e_{n}$ is a positive linear operator from $L^{1}[0,1]$ to $c_{0}$, yet since $\left\{f_{n}\right\}$ does not converge weakly to zero in $L^{\infty}[0,1]$ (any convex combination of the functions $f_{n}$ has norm 1 in $\left.L^{\infty}[0,1]\right)$ we see that $T^{*}$, hence $T$, is not weakly compact.

\section{REFERENCES}

1. J. Bourgain, Dunford-Pettis operators on $L^{1}$ and the Radon-Nikodym property, Israel J. Math. 37 (1980), 34-47.

2. - A characterization of non-Dunford-Pettis operators on $L^{1}$, Israel J. Math. 37 (1980), $48-53$.

3. N. Dunford and B. J. Pettis, Linear operators on summable functions, Trans. Amer. Math. Soc. 47 (1940), 323-392.

4. N. Dunford and J. Schwartz, Linear operators. I, Interscience, New York, 1963.

5. N. Gretsky and J. Ostroy, Thick and thin market non-atomic exchange economies, Advances in Equilibrium Theory, Lecture Notes in Economics and Mathematical Systems, No. 244, 1985, pp. 107-130.

6. _ The compact range property and $c_{0}$, Glasgow Math. J. 28 (1986), 113-114.

7. _ Dunford-Pettis operators and average range (preprint).

8. A. Grothendieck, Sur les applications lineaires faiblement compactes d'espaces du type $C(K)$, Canad. J. Math. 5 (1953), 129-173.

9. J. Holub, A note on Dunford-Pettis operators, Glasgow Math. J. 29 (1987), 271-273.

10. J. Lindenstrauss and L. Tzafriri, Classical Banach spaces, Springer-Verlag, Berlin and New York, 1973.

11. K. Musial, An operator characterization of Banach spaces which do not contain any isomorphic copy of $l^{1}$, Aarhus Universität Math. Inst. Preprint Series, No. 28, 1976/77.

12. H. Rosenthal, Convolution by a biased coin, The Altgeld Book 1975/76, Univ. of Illinois, Functional Analysis Seminar.

13. E. Saab, On Dunford-Pettis operators, Canad. Math. Bull. 25 (1982), 207-209.

14. _, On Dunford-Pettis operators that are Pettis representable, Proc. Amer. Math. Soc. 85 (1982), 363-365.

15. I. Singer, Bases in Banach spaces. I, Springer-Verlag, New York-Heidelberg-Berlin, 1970.

16. M. Talagrand, Pettis integral and measure theory, Mem. Amer. Math. Soc. no. 307, Providence, R.I., 1984.

Department of Mathematics, Virginia Polytechnic institute and State UNIVERSITY, BLACKSBURG, VIRGINIA 24061 